\title{
SEEING FROM THE USERS' EYES: AN OUTLOOK TO VIRTUAL-REALITY BASED EMPATHIC DESIGN RESEARCH
}

\author{
Hu, Xinhui; \\ Nanjappan, Vijayakumar; \\ Georgiev, Georgi V.
}

University of Oulu

\begin{abstract}
The extent to which designers can understand users often determines the quality of design outcomes. A deep understanding of users allows the designers to focus on the right problem and make optimal design decisions, which encouraged designers to empathize with users. However, the current imagination-based empathizing strategy appears to be too susceptible to their previous experience and knowledge, which has been questioned concerning effectiveness and accuracy. On the other hand, Virtual Reality (VR) technology provides an opportunity for designers to gain experience-driven empathy by immersing them in a virtual environment that mimics the users' surroundings as if they are seeing the world from users' eyes. While abundant studies covered empathy VR and empathy for design, limited attention has been paid to the chance of bringing VR, empathy, and design research together. Addressing this gap, this study explored literature across domains, identified major concerns about this approach, synthesized the evidence, and discussed the feasibility and validity of the VRbased empathic design research approach.
\end{abstract}

Keywords: Virtual reality, Human behaviour in design, Experience design, Empathy, User centred design

\author{
Contact: \\ $\mathrm{Hu}$, Xinhui \\ University of Oulu \\ Center for Ubiquitous Computing \\ Finland \\ xinhui.hu@oulu.fi
}

Cite this article: Hu, X., Nanjappan, V., Georgiev, G. V. (2021) 'Seeing from the Users' Eyes: An Outlook to VirtualReality Based Empathic Design Research', in Proceedings of the International Conference on Engineering Design (ICED21), Gothenburg, Sweden, 16-20 August 2021. DOI:10.1017/pds.2021.521 


\section{INTRODUCTION}

From the designer's perspective, users' minds work as a complex black box system whose internal mechanisms are hidden and can only be reasoned based on the inputs and outputs. Therefore, designers invest considerable effort in probing users' concerns and needs, instead of directly taking their answers as the design goal. The information gathered from inquiries with users was proven to be inadequate because important contextual, affective, and social factors and latent needs are difficult to include in user responses (Koskinen et al., 2003; Leonard and Rayport, 1997; McDonagh and Thomas, 2010). Thus, an empathic design approach was proposed that advocated designers gain a deeper understanding of users and empathise with them. However, owing to the opaqueness of the human mind, the empathising process is essentially an inference based on the designer's knowledge about users within their own mind frame, which inevitably leaves space for bias. Recent studies have questioned the accuracy of empathy and its impact on design outcomes because of the bias induced by the subjective and imaginary elements of the traditional empathising strategy (Chang-Arana et al., 2020; Robertson and Allen, 2018). Thus, designers will need a new approach that reliably understands and empathises with users, which allows them to detect both salient and latent needs. Providing an experience-driven empathetic strategy, immersive virtual reality (VR) has gained the attention of researchers as an ideal candidate for this purpose.

All major features of immersive VR, particularly its highly immersive and interactive virtual environment, suggest its significant potential in working as a simulator of the user's world. On the one hand, a completely controlled virtual environment enables designers to replicate the user's surroundings, mimic real-world sensory input, and precisely measure the interactions of the operators with VR (Bombari et al., 2015; Hu et al., 2020). On the other hand, the engagement of body movement and multimodal perception can provide much richer information than user narrations. The first-person perspective interaction and use of avatars make interactive experiences more vivid (Herrera et al., 2018). In this way, designers will have the chance to relive episodes of the users' lives through virtual avatars with the users' identities and relate these experiences to the emotion and motivation underlying their narratives. The proven effectiveness of VR in enhancing the empathetic understanding of unfamiliar communities (Herrera et al., 2018) and reducing biased tags and stereotypes (Yee and Bailenson, 2006) set an encouraging theoretical basis for this approach. However, beyond this, the discussion and validation concerning its feasibility and appropriateness are scattered across a wide range of domains, making the exploration of this complex and multi-faceted topic even more difficult.

Indeed, regarding the strategy of implementing empathy VR for design user research, there are too many questions to ask, with only a few answers being readily available. For example, what types of empathy will be elicited by the virtual experience, and how will it influence the design decisions? How accurate will it be? Can designers synchronise the identities between their body schema and avatar, particularly when they are different? Will the social rules apply between designers and avatars? More importantly, will such an enhanced empathetic understanding eventually transform into design success. The answers are either unknown or scattered across a vast range of domains and are therefore not readily available. The few existing studies on this topic are in an early exploratory stage, in which only the most salient opportunities and uncertainties are defined. Aiming to take a step further, by critically reviewing the relevant research contained in mainstream/major databases, especially Scopus and Web of Science, this study identified significant concerns regarding the opportunity to implement VR in empathic design user research and discovered evidence that contributed to validating or disproving this approach. Thus, based on the synthesis of these insights, this study presented the following: (1) what can be validated, (2) what needs to be tolerated, and (3) what needs to be tested concerning the VR-based empathic design research approach.

\section{EMPATHY, DESIGN, AND VR}

\subsection{Empathy}

Empathy is a complex mental mechanism that encompasses a broad range of phenomena and involves multiple distinctive brain functions. Depending on the domains, empathy definitions vary from a personality trait, i.e., an individual's ability, to the reaction of observing another's experiences (Bošnjaković and Radionov, 2018; Yagil, 2015). Among these domain-specific views, such a perspective has been commonly shared: empathy refers to the ability to emotionally feel and 
intellectually understand others in their particular situations (Bošnjaković and Radionov, 2018; Herrera et al., 2018; Yagil, 2015).

In a certain sense, this view echoes the prevalent dichotomy between the affective and cognitive aspects of empathy. Respectively described as "I feel what you feel" and "I understand what you feel", affective empathy and cognitive empathy rely on two distinctive neural mechanisms (Shamay-Tsoory et al., 2009). The former is a state of emotion that resonates with others and involves human primitive and automatic emotional functions, whereas the latter is a deliberate and rational perspective-taking process that engages advanced cognitive functions (Banakou et al., 2013; Bošnjaković and Radionov, 2018; Davis et al., 1996; Decety, 2011; Eres, 2016; Shamay-Tsoory et al., 2009). Moreover, some neuroscience studies have suggested that, based on brain activity, more types of empathy can be defined, such as somatic empathy, compassionate empathy, and emotion regulation (Bernhardt and Singer, 2012; Decety, 2011; Eres, 2016; Powell and Roberts, 2017; Price and Dambha-Miller, 2019; Yagil, 2015). However, affective empathy and cognitive empathy are still central to the research on this phenomenon.

\subsection{Empathy in Design}

Empathy was initially introduced in the design to mitigate the mind gaps of designers in user research. Users are not omniscient regarding their situations, concerns, and needs. Their unaware and latent needs will be constantly absent from their answers, and hence cannot be gained through inquiry-based methods (Leonard and Rayport, 1997). Thus, even the best-designed questions and the frankest user cannot guarantee an accurate understanding. To address this issue, an empathic design approach was proposed, which advocates designers not only to know about the users but also to be emotionally related to them (Kouprie and Visser, 2009). More specifically, this approach encourages designers to gain an in-depth understanding of users through immersive observations in the field (Leonard and Rayport, 1997) and then construct a pseudo-user perspective based on the insights gained (Kouprie and Visser, 2009). In this way, designers have the chance to set aside their own mindsets and attempt to bridge the empathetic gap by imagining such simulated users' perspectives (Koskinen et al., 2003; Kouprie and Visser, 2009; McDonagh and Thomas, 2010).

From a practical perspective, however, the empathic design approach has an inherent risk of bias owing to human-related errors. This approach is essentially an information collection- and imagination-based empathising process focusing on the design elements, which involves many subjective mental processes and leaves significant space for information distortion. Moreover, the existence of the observer is often obtrusive enough to alternate human behaviours, as shown by the Hawthorne effect. Perhaps owing to these inevitable issues, the empathic design approach seemed to be adopted more often for inspirational purposes than for informative purposes. For example, when Leonard and Rayport first proposed this approach in 1997, the observation stage was followed by a data-capturing stage as a supplement. In more recent studies, this approach is also appreciated in the sense of allowing designers to develop new ways of "seeing, thinking, and experiencing" (McDonagh and Thomas, 2010). The expectation of this method centred on its guidance of user research, rather than the correctness of the imagination and perspectivetaking, owing to the inevitable human-related bias. Even so, recent studies on empathy accuracy and design outcomes questioned this method, and its result indicated that the effort to empathise with users did not bring a comparable effect, whereas enhanced empathy can bias the design decisions (ChangArana et al., 2020; Robertson and Allen, 2018). If further evidence complies with this study, the effectiveness of this approach may be challenged.

In an empathic design approach, the dilemma is that the right problem to solve has been defined, but a suitable apparatus is absent. Fortunately, promising virtual reality technology demonstrates the potential of such an apparatus.

\subsection{VR for Empathy}

The affordances of virtual reality (VR) technology make it an ideal instrument for studying empathy. VR has the capability to be a simulator of the real world while supporting the immersive and multisensory interaction of the operators. By replicating one's surroundings, body features, and perceptual input in a virtual environment, VR allows its operators to briefly but vividly live an episode of another person's life. Previous literature suggests that VR can reshape empathy in three dimensions: recognition, emotion, and behaviour (Liu, 2020), which echoes the cognitive and affective aspects of empathy and consequent actions. Because gaining similar experiences may be the most straightforward way to build empathy between people, VR seems to be the perfect medium for eliciting empathy. Therefore, this technology is often depicted as an ultimate empathy machine (Herrera et al., 2018; Liu, 2020; Ventura et al., 2020). 
Studies across domains have reported an enhanced empathy level among unfamiliar groups with VR. For example, Herrera et al. (2018) compared perspective-taking tasks to home-less people with different immersion levels. They found that the VR condition demonstrated the most robust impact and more behavioural change through empathy. The inclusion of virtual avatars in a VR-based empathising approach also facilitates empathy (Ahn et al., 2013; Yee and Bailenson, 2007). An interesting instance is from a study by Yee and Bailenson. Based on this study, when participants are embodied in an avatar with extreme outgroup member features, a short virtual interaction can change a person's negative stereotypes (2006). Schutte and Stilinović (2017) also found that VR experience results in a greater engagement and a higher empathy level than non-immersive media. More studies have shown that immersive perspective-taking experiences with VR enhance empathy and understanding of people with different identities (Gillath et al., 2008). However, as the themes of many empathy VR projects relate to the lives of suffering people, such as adolescents during war and homeless people (Bujić et al., 2020; Herrera et al., 2018), some researchers have questioned whether the VR experience provokes compassionate feelings or real empathy (Bollmer, 2017; Jones, 2017; Laws, 2020). In general, the results of meta-analyses concluded that VR experience has a positive influence on empathy, although the estimation of the effect size varies (Laws, 2020; Liu, 2020).

\subsection{VR-based Empathic Design}

Because their features complement each other, there is an opportunity to bring VR, empathy, and design research together. In this way, some of their limitations can be offset by each other, while new opportunities emerge. For example, the VR-based empathising approach allows designers to detour from the primary source of bias in an empathic design. Similarly, studying empathy VR in the context of design enables participants to demonstrate more natural behaviours because their attention will be occupied by the design tasks and spare less on behaviour adjustments (Lam, 2015). Moreover, previous studies have shown that operators can synchronise both motion and perception with their embodied avatars. Viewing the avatar in a virtual mirror with the same actions allows operators to develop an illusion of owning the avatar as a part of their bodies (Loon et al., 2018), which can activate an operator's motor-sensory and embodied cognition and enrich the information perceived by the designers. Furthermore, as shown by Banakou et al.(2013), embodying adults in the avatars of 4-year-old children have influenced their estimation of the object size, which suggests that operator perception can match the features of their avatars. These findings imply that when designers embody themselves into avatars with the characteristics of the target users, both their visceral intuitive perception and the higher-level cognitive process may be reshaped by the features of the avatar. If such an effect can be validated, it means that a VR-based empathic design approach can break the empathetic wall between users and designers. Because they can precisely exclude and add variables to the virtual environment, it is also noteworthy that designers can strike a balance between standardisation and high ecological validity (Gillath et al., 2008).

However, new challenges also arise. First, what has been known and what remains unknown regarding implementing VR in empathic design research has been nebulous. What will be necessary to validate or disprove this approach? In addition, what questions have already been answered? Finally, what uncertainties require further investigation? Answers to these questions are unavailable. If the designers want to examine the feasibility of this approach, there will be no anchoring point to initiate such research. However, some phenomena that were viewed as harmless to other research fields may have an unexpected impact on the design process. For example, the self-other merging phenomenon, which refers to perceiving others' thoughts as more self-like owing to enhanced empathy, is usually considered beneficial and prosocial. But it seems to be a sign of blurring the identity of users and designers (Hu and Georgiev,2020). Similarly, the Proteus effect, i.e., changes in behaviour and attitude owing to a preconception of the identity of the embodying avatar, is considered to influence the operator's behaviours in extremely subtle ways (Ratan, 2013). Yet this phenomenon can be a source of bias for designers because it is often explained as a priming effect or as externalised stereotypes (Ash, 2016). These newly induced uncertainties need to be specified. To date, only a few studies have discussed relevant topics and identified several salient uncertainties concerning this approach. A further step toward understanding VR-based empathic design research is needed. Addressing this unmet need, this study explored the major concerns regarding this approach and synthesised the information in a structural manner, as shown in Figure 1. 


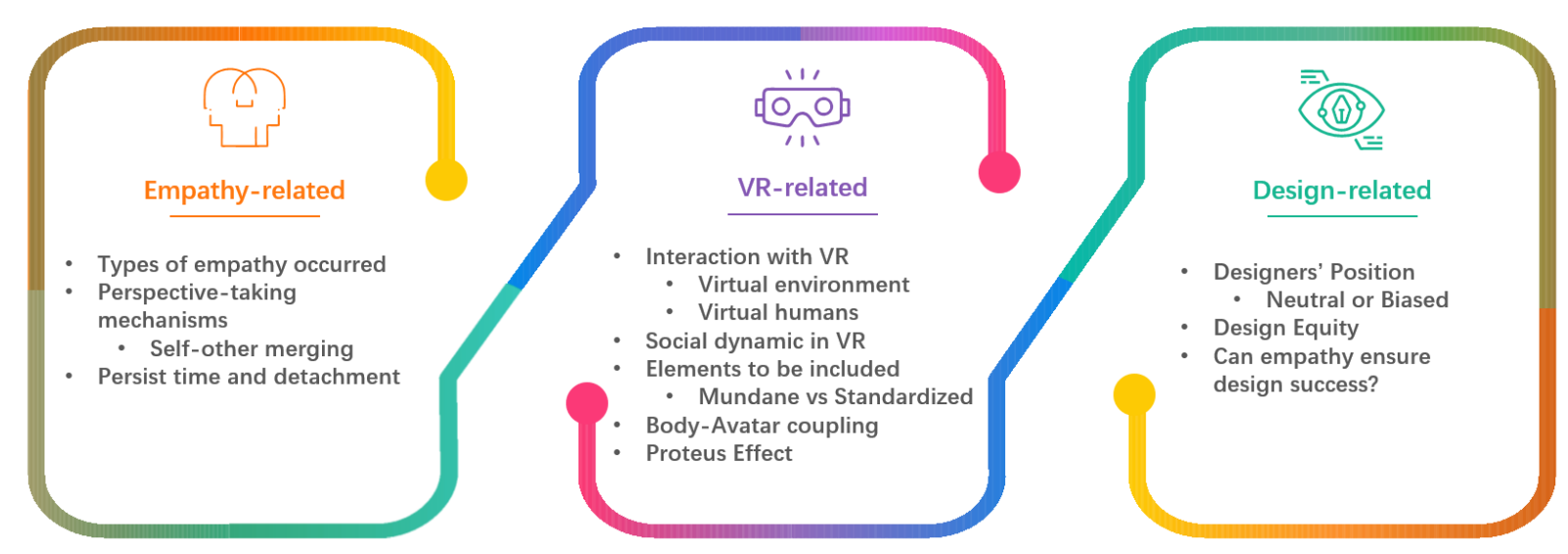

Figure 1. Concerns about VR-based empathic design research

Based on their focus, these concerns were grouped into three categories for further exploration.

- Empathy-related concerns: These concerns involve underlying mental mechanisms and their potential consequences. For example, what types of empathy will occur during this process? What perspective-taking strategies are adopted? Will the succeeding self-other merging induce bias to designers? How long will enhanced empathy persist? Will designers be able to detach from their empathetic status to users and return neutral?

- Design-related concerns: Design-related concerns concentrate on the risk of bias in the position. Because VR has a more substantial effect in enhancing empathy, designers will deviate from the neutral position and become overly aligned with users. Will the deepened knowledge facilitate design equity, particularly for peripheral user groups? Moreover, will empathy necessarily be transferred into design success?

- VR-related concerns: This type of concern is mostly about the details of the human-VR interaction and the body-avatar coupling issue. To what extent should a virtual environment mirror the user's surroundings? Can virtual humans reliably simulate interpersonal interactions around users? Will the social interaction rules, such as the Hawthorne effect, still apply in the virtual world? How will body schema transfer during body-avatar coupling impact the identity recognition of the designer?

Then, targeting the identified uncertainties, a second literature review was conducted to collect evidence that contributes to validating or disproving this approach. This second review stems from the previously identified concerns, with an emphasis on empirical evidence. Based on the results, the gained insights are presented in three sections: (1) what can be validated, (2) what needs to be tolerated, and (3) what needs to be further tested.

\section{WHAT HAS BEEN VALIDATED}

- Interaction with virtual human agent is comparable to a real human

In general, previous studies have suggested that the interaction of operators with virtual human-like agents can be comparable to their interaction with a real human (Bombari et al., 2015; Gillath et al., 2008; Heyselaar et al., 2017). More specifically, depending on the attention spared to the virtual partner, operators are able to calibrate their perception and reaction to the virtual agents. When interactions with virtual humans engage in intensive attention and higher-level cognitive processes, operators tend to demonstrate the same behaviour traits as interacting with real humans. For example, Heyselaar et al. (2017) found that a human-like virtual agency can elicit the same syntactic priming effect during a language task, while a computer-like agency did not show this effect. Moreover, multiple studies have reported that giving speeches to virtual audiences can induce similar anxious reactions as when facing a real audience (Bombari et al., 2015).

By contrast, when virtual humans undertake the role of bystanders, operators may react to them both rationally as computer agents and unconsciously as people. The presence of virtual humans has been widely observed to provoke automatic visceral responses similar to those of real humans. Bailenson et al. (2003) found that operators managed interpersonal distance during mutual gaze with virtual humans in the same manner as in real social life. The social inhibition effect, which refers to the worse performance and higher arousal in physiological responses when having other people around, also applies when in the presence of virtual humans (Bombari et al., 2015). However, when deliberately asked about the 
perception of the virtual humans, the operators often reported that they did not perceive the virtual humans as observers (Garau et al., 2005) and sometimes forgot the virtual humans completely when focusing on the task (Lam, 2015). These results suggest that virtual humans can reliably elicit the same automatic responses as in real-world scenarios without being perceived as obtrusive. Thus, using the virtual environment and virtual humans for designer user research is a valid approach.

- Social interaction rules apply to the virtual environment in most cases

In addition to the social inhibition effect and interpersonal distance management strategy discussed above, most other real-world social dynamics apply in the virtual world, although sometimes with subtle differences. For example, the implicit rules in social interaction, such as a 'firm gaze increasing persuasiveness,' 'repeating other's movements and bringing a positive impression', and 'negative feedback inducing anxiety', have all been observed in the interactions with virtual humans (Bailenson et al., 2003; Bombari et al., 2015). Furthermore, scenarios involving complex social behaviours and cognition can also be reproduced in the virtual world. Slater et al. (2006) were able to repeat Milgram's electric shock obedience experiment with virtual humans. Although the human participants completely understood that both the virtual human agents and the electric shock were fake, their responses echoed the original results of the experiment at the subjective, behavioural, and physiological levels (Bombari et al., 2015; Slater et al., 2006). Moreover, it is interesting that the Hawthorne effect and other observerinduced impacts in social interactions seem to apply to a virtual world without being consciously realised by the operators. The operators always report not perceiving virtual humans as real people, whereas their physiological and behavioural traits indicate the opposite (Bombari et al., 2015; Garau et al., 2005; Lam, 2015). Thus, it seems that human instinctive and conscious responses to social scenarios, which are usually intertwined with each other, can be observed separately in VR-based interactions. This possibility sheds new light on behavioural studies in a design and in a broader context.

\section{WHAT NEEDS TO BE TOLERATED}

- $\quad$ Self-other merging: Blurring the boundaries between self and other

Self-other merging is a phenomenon that naturally occurs in the active perspective-taking process, which is featured by perceiving others' thoughts and feelings as more "self-like" (Davis et al., 1996) with an enhanced willingness for helping people (Ahn et al., 2013; Herrera et al., 2018). More specifically, this is a projection and ascription of positive self-relevant traits to others (Ahn et al., 2013; Davis et al., 1996), which seems to blur the boundaries of one's own identity with that of another (Devecchi and Guerrini, 2017). Compared to the effortful imagination-based perspective-taking process, the VR-based perspective-taking task, offering a more vivid experience, brings a more salient self-other merging effect (Herrera et al., 2018). In most cases, this phenomenon is harmless and even favourable, as it is usually accompanied by increases in empathy and prosocial behaviours.

However, blurring the boundaries between the self and others can induce severe bias in framing the design problem and making design decisions. Because self-other merging is an inevitable by-product of the perspective-taking process, designers can only tolerate this phenomenon while paying attention to controlling its impact within an acceptable range. The extent of self-other merging is usually measured using questionnaires and scales. For example, Davis et al. (1996) used a questionnaire about help intention to detect self-other merging, whereas Ahn (2013) used the Inclusion of Other in the Self Scale' and the Implicit Attitude Test (IAT) for testing. Nonverbal behavioural measurements, such as behavioural coding, are necessary, as there were cases where significant differences were detected, while the self-report showed no differences (Herrera et al., 2018).

\section{- $\quad$ Proteus Effect: Preconception of avatar's identity biases real-world attitude and behaviour}

The Proteus effect refers to shifts in the operator's attitudes and behaviours in accord with the identity features derived from their embodied avatars (Ahn et al., 2013; Reinhard et al., 2020; Yee and Bailenson, 2007). In other words, VR operators demonstrate the behaviours and attitudes that they subjectively believed to align with the embodying avatar. When defining this phenomenon, Yee and Bailenson proposed two potential explanations: self-perception theory and the social identity model of deindividuation effects theory (2007). The former claimed that the phenomenon was due to operators using their self-observations as a reference frame for behaviour and are hence susceptible to the avatar features, whereas the latter argued that this was because the features of the avatar become the dominant identity cue and therefore lead operators to reinforce the salient behaviours related to the group. Other alternative explanations include the priming effect based on avatar appearance and externalised 
stereotypes (Ash, 2016). However, in either explanation, the Proteus effect is purely derived from the operator's own expectations and assumptions of the avatar's identity, which is inherently biased.

IAT was proven effective in measuring the attitude shift due to the Proteus Effect (Peck et al., 2013), while behavioural changes are often measured indirectly. For example, Yee and Bailenson (2003) detected the existence of this phenomenon by measuring how an avatar's attractiveness and height influence the operator's interpersonal distance management. Peña et al. (2009) and de Rooji et al. (2017) observed differences in aggression and creativity by alternating the clothes of avatars. There seem to be no attempts to quantify the impact of the Proteus Effect. In the design context, the Proteus Effect can potentially be measured by setting pre-VR and post-VR design tasks and comparing the rationale of design outcomes.

\section{WHAT NEED TO BE TESTED}

\section{1 mpathy-related concerns}

- What types of empathy occur during this process and how do they occur?

As mentioned in the earlier section, there are multiple types of empathy mediated by different neural pathways that can evoke different physiological responses. The existing analysis suggests that both cognitive and affective empathy occur in interactions with VR (Herrera et al., 2018; Loon et al., 2018). Beyond this, little is known, probably because the results do not impact the effectiveness of this approach. However, for research design, knowing which types of empathy occur in what sequence and how long their impacts persist will help track the empathetic strategy in the design. Physiological signals, such as heart rate and galvanic skin response (Bombari et al., 2015), are commonly used to track emotion-related mechanisms. Since cognitive empathy primarily activates the prefrontal and temporal lobes, while affective empathy activates the inferior frontal gyrus and insula, brain imaging techniques such as electroencephalography (EEG) and functional near-infrared spectroscopy (fNIRS) may allow designers to detect the difference.

- What is the perspective-taking strategy during avatar embodiment?

Humans can adopt two perspective-taking strategies: imagine-self and imagine-other (Batson et al., 1997). In the former case, people imagine feeling as if they were the observed person in that situation. By contrast, in the latter case, people imagine how the observed person perceives and feels in their position. The imagine-other strategy tends to evoke mental representations of the observed person and altruistic motivation for prosocial behaviours, whereas the imagine-self approach triggers self-related knowledge, personal stress, and egocentric motivation to help (Batson et al., 1997; Davis et al., 1996). The situation may become complicated when operators undertake perspective-taking tasks when embodying an out-group avatar, particularly in the design context. When designers embody the avatar of users, what will be their perspective-taking strategy? Will they perceive themselves as users, imagine themselves as users, or neither? The answer can help designers detect and offset potential biases. The perspective-taking strategy can be measured with physiological methods, as it has been reported that, when viewing painful experiences, the imagine-other strategy brings about a vessel contraction, whereas the imagine-self strategy leads to palm sweat and feelings such as tension and nervousness (Batson et al., 1997).

- How long will the empathy persist? Can the operator detach from the status?

The enhanced empathy level owing to the interaction with VR can persist from a day (Ahn et al., 2013) to weeks (Herrera et al., 2018), based on the observation of the participant's prosocial behaviours. It is unknown whether operators can detach from an empathetic status. In Kouprie and Visser's empathy model (2009), a detachment stage was included that allowed designers to quit from the resonance with users and step back into their original role; however, its effectiveness has not been assessed. Further investigation and a better measurement apparatus are required.

\subsection{R-related concerns}

- $\quad$ To which extent should the virtual environment replicate the real world?

This question is a less critical concern because it is still limited by the currently available technology. However, when VR technology eventually achieves its full potential for a high-fidelity simulation of the physical world, designers will need to determine the extent to which the virtual world should replicate the real world. Including every detail in the real world inside the virtual environment is infeasible and unnecessary. Trying to determine which element is important is also risky, as it is not rare for seemingly 
irrelevant factors to be found to play an important role. The contextual elements to be included and excluded from the virtual environment must be selected carefully, and a guideline should be established.

- $\quad$ Can operators synchronise identity with the embodied avatars?

When embodying an avatar with a different identity, how do the operators process the conflicts? Will the operator synchronise their identity with the avatar? While positive attitude shifts have been observed, it is unclear whether this results from the synchronised identity, the enhanced empathy, the elicited compassionate feeling, the Proteus Effect, or a mixture of them. These mental processes are intertwined with each other and are difficult to define. A potential method to address this issue is to use pre-VR and post-VR design tasks to determine what identity was held from the design outcomes.

\subsection{Design-related concerns}

- Can designers retain a neutral perspective in VR-based empathy design research?

In this case, the concern is not whether designers can stay in a neutral position, but how the benefits of enhanced empathy and bias imposed by the VR experience trade off each other. Although more investigations are needed, the strong emotional connection between operators and avatars seems to be problematic.

- Will VR-based user research enhance design equity?

It is difficult to answer this question before VR is implemented for design user research. Coleman et al. (2003) suggested that inadequate empathy in the design process will lead to an over-focus on the functional aspects while ignoring the emotional and reflective needs, which can be overcome with empathy VR. By contrast, Robertson and Allen (2018) claimed that empathy would lead to unbalanced attention to the designers' features and leave other aspects less concerned. The answers can only be found in actual practice.

- Will empathy necessarily lead to design success?

This question is essentially about whether empathy guarantees a deeper understanding and whether those understandings guarantee a better design that meets user needs. The critical issue in this case is how accurate empathy is. Recent challenges in empathic design have focused on the accuracy of empathy. Thus, to find the answer, the truth underlying the other identified uncertainties regarding the validity of an empathic design needs to first be investigated.

\section{CONCLUSION}

In conclusion, this study identified the opportunity to facilitate design user research with empathy VR, determined the significant concerns related to this approach, and explored the answers to such concerns. This study of validated that VR is a suitable medium for simulating user scenarios and identified two inevitable sources of bias when using a VR-based empathic design research approach: the self-other merging effect and the Proteus effect. Moreover, this study has synthesised the uncertainties to be investigated in this line of work while specifying the potential methods for testing. In the next step, we plan to carry out a series of empirical studies to further explore the unanswered questions identified here, focusing on opportunities to offset the potential biases and uncertainties that accompany VR use.

\section{ACKNOWLEDGMENTS}

This research has been financially supported by Academy of Finland 6 Genesis Flagship (Grant 318927)

\section{REFERENCES}

Ahn, S., Le, A. and Bailenson, J. (2013), "The Effect of Embodied Experiences on Self-Other Merging, Attitude, and Helping Behavior", Media Psychology, Routledge, Vol. 16 No. 1, pp. 7-38. 10.1080/15213269.2012.755877

Ash, E. (2016), "Priming or Proteus Effect? Examining the Effects of Avatar Race on In-Game Behavior and PostPlay Aggressive Cognition and Affect in Video Games", Games and Culture, SAGE Publications, Vol. 11 No. 4, pp. 422-440. https://doi.org/10.1177/1555412014568870

Bailenson, J., Blascovich, J. and Beall, A. (2003), "Interpersonal Distance in Immersive Virtual Environments", Personality \& Social Psychology Bulletin, Vol. 29, pp. 819-33. https://doi.org/10.1177/0146167203029007002

Banakou, D., Groten, R. and Slater, M. (2013), "Illusory ownership of a virtual child body causes overestimation of object sizes and implicit attitude changes", Proceedings of the National Academy of Sciences, National Academy of Sciences, Vol. 110 No. 31, pp. 12846-12851. https://doi.org/10.1073/pnas.1306779110 
Batson, C.D., Early, S. and Salvarani, G. (1997), "Perspective Taking: Imagining How Another Feels Versus Imaging How You Would Feel", Personality and Social Psychology Bulletin, Vol. 23 No. 7, pp. 751-758. https://doi.org/10.1177/0146167297237008

Bernhardt, B.C. and Singer, T. (2012), “The Neural Basis of Empathy”, Annual Review of Neuroscience, Vol. 35 No. 1, pp. 1-23. https://doi.org/10.1146/annurev-neuro-062111-150536

Bollmer, G. (2017), "Empathy machines”, Media International Australia, Vol. 165 No. 1, pp. 63-76. https://doi.org/10.1177/1329878X17726794

Bombari, D., Schmid Mast, M., Canadas, E. and Bachmann, M. (2015), "Studying social interactions through immersive virtual environment technology: virtues, pitfalls, and future challenges", Frontiers in Psychology, Frontiers, Vol. 6. https://doi.org/10.3389/fpsyg.2015.00869

Bošnjaković, J. and Radionov, T. (2018), "Empathy: Concepts, Theories and Neuroscientific Basis”, Alcoholism and Psychiatry Research: Journal on Psychiatric Research and Addictions, Vol. 54 No. 2, pp. 123-150. https://doi.org/10.20471/dec.2018.54.02.04

Bujić, M., Salminen, M., Macey, J. and Hamari, J. (2020), “'Empathy machine’: how virtual reality affects human rights attitudes", Internet Research, Emerald Publishing Limited, Vol. 30 No. 5, pp. 1407-1425. https://doi.org/10.1108/INTR-07-2019-0306

Chang-Arana, Á.M., Piispanen, M., Himberg, T., Surma-aho, A., Alho, J., Sams, M. and Hölttä-Otto, K. (2020), "Empathic accuracy in design: Exploring design outcomes through empathic performance and physiology", Design Science, Cambridge University Press, Vol. 6. https://doi.org/10.1017/dsj.2020.14

Coleman, R., Lebbon, C. and Myerson, J. (2003), "Design and empathy”, in Clarkson, J., Keates, S., Coleman, R. and Lebbon, C. (Eds.), Inclusive Design: Design for the Whole Population, Springer, London, pp. 478-499. https://doi.org/10.1007/978-1-4471-0001-0_29

Davis, M.H., Conklin, L., Smith, A. and Luce, C. (1996), "Effect of perspective taking on the cognitive representation of persons: A merging of self and other", Journal of Personality and Social Psychology, Vol. 70 No. 4, pp. 713-726. https://doi.org/10.1037/0022-3514.70.4.713

Decety, J. (2011), "Dissecting the Neural Mechanisms Mediating Empathy", Emotion Review, Vol. 3 No. 1, pp. 92-108. https://doi.org/10.1177/1754073910374662

Devecchi, A. and Guerrini, L. (2017), "Empathy and Design. A new perspective", The Design Journal, Vol. 20 No. sup1, pp. S4357-S4364. https://doi.org/10.1080/14606925.2017.1352932

Eres, R. (2016), "Scanning for empathy", Australasian Science, Vol. 37 No. 2, p. 30.https:// doi/10.3316/INFORMIT.146323167516241

Garau, M., Slater, M., Pertaub, D.-P. and Razzaque, S. (2005), "The Responses of People to Virtual Humans in an Immersive Virtual Environment", Presence: Teleoperators and Virtual Environments, MIT Press, Vol. 14 No. 1, pp. 104-116. https://doi.org/10.1162/1054746053890242

Gillath, O., McCall, C., Shaver, P.R. and Blascovich, J. (2008), "What Can Virtual Reality Teach Us About Prosocial Tendencies in Real and Virtual Environments?”, Media Psychology, Vol. 11 No. 2, pp. $259-282$. https://doi.org/10.1080/15213260801906489

Herrera, F., Bailenson, J., Weisz, E., Ogle, E. and Zaki, J. (2018), "Building long-term empathy: A large-scale comparison of traditional and virtual reality perspective-taking", PLOS ONE, Vol. 13 No. 10, p. e0204494 https://doi.org/10.1371/journal.pone.0204494

Heyselaar, E., Hagoort, P. and Segaert, K. (2017), "In dialogue with an avatar, language behavior is identical to dialogue with a human partner", Behavior Research Methods, Vol. 49 No. 1, pp. 46-60. https://doi.org/10.3758/s13428-015-0688-7

Hu, X., Georgiev, G.V. and Casakin, H. (2020), "Mitigating Design Fixation with Evolving Extended Reality Technology: An Emerging Opportunity", Proceedings of the Design Society: DESIGN Conference, Cambridge University Press, Vol. 1, pp. 1305-1314. https://doi.org/10.1017/dsd.2020.91

Hu, X., \& Georgiev, G. V. (2020). Opportunities with Uncertainties: The Outlook of Virtual Reality in the Early Stages of Design. Proceedings of the Sixth International Conference on Design Creativity (ICDC 2020), Oulu, Finland, 215-222. https://doi.org/10.35199/ICDC.2020.27

Jones, S. (2017), "Disrupting the narrative: immersive journalism in virtual reality", Journal of Media Practice, Routledge, Vol. 18 No. 2-3, pp. 171-185. https://doi.org/10.1080/14682753.2017.1374677

Koskinen, I., Mattelmäki, T. and Battarbee, K. (2003), Empathic Design.

Kouprie, M. and Visser, F.S. (2009), "A framework for empathy in design: stepping into and out of the user's life", Journal of Engineering Design, Vol. 20 No. 5, pp. 437-448. https://doi.org/10.1080/09544820902875033

Lam, N. (2015), Investigating the Impact of Virtual Actors in Priming on Presence in Virtual Reality, Master of Science (Human-Computer Interaction with Ergonomics), University College London.

Laws, A.L.S. (2020), “Can Immersive Journalism Enhance Empathy?”, Digital Journalism, Routledge, Vol. 8 No. 2, pp. 213-228. https://doi.org/10.1080/21670811.2017.1389286

Leonard, D. and Rayport, J. (1997), "Spark Innovation Through Empathic Design”, Harvard Business Review, Vol. 75, pp. 102-13. 
Liu, Y. (2020), "The application of virtual reality in empathy establishment: Foresee the future", presented at the 2020 5th International Conference on Computational Intelligence and Applications (ICCIA), pp. 188-193. https://doi.org/10.1109/ICCIA49625.2020.00043

Loon, A. van, Bailenson, J., Zaki, J., Bostick, J. and Willer, R. (2018), "Virtual reality perspective-taking increases cognitive empathy for specific others", PLOS ONE, Vol. 13 No. 8, p. e0202442. https://doi.org/10.1371/journal.pone.0202442

McDonagh, D. and Thomas, J. (2010), "Disability + Relevant Design: Empathic Design Strategies Supporting More Effective New Product Design Outcomes”, The Design Journal, Vol. 13, pp. 180-198. https://doi.org/10.2752/175470710X12735884220899

Peck, T.C., Seinfeld, S., Aglioti, S.M. and Slater, M. (2013), "Putting yourself in the skin of a black avatar reduces implicit racial bias", Consciousness and Cognition, Vol. 22 No. 3, pp. 779-787. https://doi.org/10.1016/j.concog.2013.04.016

Peña, J., Hancock, J.T. and Merola, N.A. (2009), “The Priming Effects of Avatars in Virtual Settings”, Communication Research, SAGE Publications Inc, Vol. 36 No. 6, pp. 838-856. https://doi.org/10.1177/0093650209346802

Powell, P.A. and Roberts, J. (2017), "Situational determinants of cognitive, affective, and compassionate empathy in naturalistic digital interactions", Computers in Human Behavior, Vol. 68, pp. 137-148. https://doi.org/10.1016/j.chb.2016.11.024

Price, A. and Dambha-Miller, H. (2019), "Empathy as a state beyond feeling: a patient and clinician perspective", Journal of the Royal Society of Medicine, Vol. 112 No. 2, pp. 57-60. https://doi.org/10.1177/0141076818790665

Ratan, R. (2013), "Self-Presence, Explicated: Body, Emotion, and Identity Extension into the Virtual Self", Handbook of Research on Technoself: Identity in a Technological Society, IGI Global, pp. 322-336. https://doi: 10.4018/978-1-4666-2211-1.ch018

Reinhard, R., Shah, K., Faust-Christmann, C. and Lachmann, T. (2020), “Acting your avatar's age: effects of virtual reality avatar embodiment on real life walking speed", Media Psychology, Routledge, Vol. 23 No. 2 , pp. 293-315. https://doi.org/10.1080/15213269.2019.1598435

Robertson, R. and Allen, P. (2018), "Empathy Is not Evidence: 4 Traps of Commodified Empathy", Ethnographic Praxis in Industry Conference Proceedings, Vol. 2018 No. 1, pp. 104-124. https://doi.org/10.1111/15598918.2018.01199

de Rooij, A., Corr, P and Jones, S. (2017), "Creativity and Emotion: Enhancing Creative Thinking by the Manipulation of Computational Feedback to Determine Emotional Intensity", Proceedings of the 2017 ACM SIGCHI Conference on Creativity and Cognition, Association for Computing Machinery, Singapore, Singapore, pp. 148-157. https://doi.org/10.1145/3059454.3059469

Schutte, N. and Stilinović, E. (2017), "Facilitating empathy through virtual reality", Motivation and Emotion, Vol. 41 No. 6, pp. 708-712. https://doi.org/10.1007/s11031-017-9641-7

Shamay-Tsoory, S.G., Aharon-Peretz, J. and Perry, D. (2009), "Two systems for empathy: a double dissociation between emotional and cognitive empathy in inferior frontal gyrus versus ventromedial prefrontal lesions", Brain, Vol. 132 No. 3, pp. 617-627. https://doi.org/10.1093/brain/awn279

Slater, M., Antley, A., Davison, A., Swapp, D., Guger, C., Barker, C., Pistrang, N., et al. (2006), "A Virtual Reprise of the Stanley Milgram Obedience Experiments", PLOS ONE, Public Library of Science, Vol. 1 No. 1, p. e39. https://doi.org/10.1371/journal.pone.0000039

Ventura, S., Badenes-Ribera, L., Herrero, R., Cebolla, A., Galiana, L. and Baños, R. (2020), "Virtual Reality as a Medium to Elicit Empathy: A Meta-Analysis", Cyberpsychology, Behavior, and Social Networking. https://doi.org/10.1089/cyber.2019.0681

Yagil, Y. (2015), “The Physiology of Empathy: Theoretical and Practical Implications”, Encountering Empathy, p. 3.

Yee, N. and Bailenson, J. (2006), "Walk a mile in digital shoes: The impact of embodied perspective-taking on the reduction of negative stereotyping in immersive virtual environments", Proceedings of PRESENCE 2006: The 9th Annual International Workshop on Presence, Cleveland, Ohio, USA.

Yee, N. and Bailenson, J. (2007), "The Proteus Effect: The Effect of Transformed Self-Representation on Behavior”, Human Communication Research, Oxford Academic, Vol. 33 No. 3, pp. 271-290. https://doi.org/10.1111/j.1468-2958.2007.00299.x 